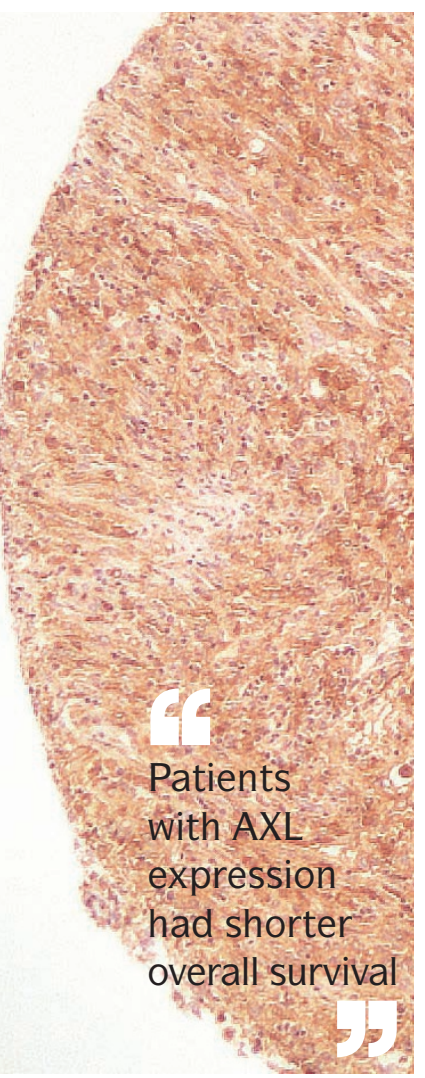

Image courtesy of Rui Reis, Barretos Cancer Hospital

\title{
AXL expression predicts prognosis
}

New data published in Urologic Oncology show that increased expression of the receptor tyrosine kinase AXL is associated with worse clinical outcomes in patients with metastatic renal cell carcinoma (mRCC) who have previously received sunitinib. Thus, AXL expression could be a prognostic biomarker for patients with this disease.

Corresponding author Rui Reis explained to Nature Reviews Urology how their research group had previously observed that AXL expression was predictive of the response of glioblastoma cells to sunitinib in vitro. As sunitinib is a treatment that is frequently given to patients with mRCC, the researchers hypothesized that AXL expression could also be a predictive biomarker in this disease. To test this hypothesis, the investigators analysed AXL expression in tumour samples from 64 patients with mRCC (51 clear cell RCC (ccRCC) and 13 of other subtypes) using immunohistochemistry. Results showed that $46.8 \%$ (30 out of 64) of samples exhibited AXL expression (43.1\% of ccRCCs and $61.5 \%$ of other subtypes). Univariate analysis revealed that AXL expression was positively associated with poor prognostic features, such as presence of metastasis and sarcomatoid histology; however, only progression on sunitinib was significantly associated $(\mathrm{P}=0.046)$, particularly for ccRCC $(\mathrm{P}=0.015)$. In total, $54.7 \%$ of the cohort progressed while receiving sunitinib. Of the patients with AXL expression and who received sunitinib, 77.8\% had disease progression, whereas $51.9 \%$ of patients who received sunitinib but were negative for AXL expression experienced disease progression. Patients with AXL expression had shorter overall survival: 19 months compared with 41 months for those without AXL expression. Multivariate analysis showed that positivity for AXL expression was associated with poor prognosis $(\mathrm{HR}=2.007)$.

"This study is the first to show that positive AXL expression is a prognostic biomarker in patients with mRCC who have received sunitinib. Moreover, AXL expression in mRCC could also be a predictive biomarker of response to new and effective antiAXL drugs, such as cabozantinib," Reis concluded.

Louise Stone

ORIGINAL ARTICLE Zucca, L. E.e tal. Expression of tyrosine kinase receptor $A X L$ is associated with worse outcome of metastatic renal cell carcinomas treated with sunitinib. Urol. Oncol. http://dx.doi.org/ 10.1016/j.urolonc.2017.09.003 (2017) 